\title{
Reducing Anxiety: Training Attentional Disengagement from Threat Using a Modified Spatial Cueing Task
}

\author{
Stacy Eltiti*, John J. Siefke, David Lomas and John Williams \\ Rosemead School of Psychology, Rosemead School of Psychology, Biola University, 13800 Biola Ave, La Mirada, CA, USA
}

Received: April 10, 2014; Accepted: April 22, 2014; Published: April 22, 2014

*Corresponding author: Stacy Eltiti, Rosemead School of Psychology, Biola University, 13800 Biola Ave, La Mirada, CA. 90639, USA, Tel: 562-903-4738. Fax: 562-903-4864.E-mai: stacy.eltiti@biola.edu

\begin{abstract}
It is well established that trait anxious individuals have an attentional bias for threatening information and stimuli, as demonstrated through both the dot probe and cueing paradigms. In recent efforts to reduce attentional biases and minimize anxiety symptomatology, variations of the dot probe task have been used to train attention away from threat. However, under this paradigm, it is uncertain what the underlying mechanism for attentional bias is. Is anxiety being perpetuated by a shift in attention to threat or does it result from one's inhibited ability to disengage from threat once it is attended to? The purpose of the present study was to train attention away from threat using a cueing task, thus isolating attentional biases to difficulties in disengaging from threat. The goal of this task was to train attention by manipulating the contingency between the location of a target and a threatening or affectively neutral pictorial cue. Eighty-nine trait anxious college students participated in this study. It was hypothesized that participants in training groups would be able to disengage from threat faster and subsequently show a greater decrease in state anxiety when compared to controls. However, the data failed to support the primary hypotheses of this study. Interestingly, none of the groups showed an attentional threat bias at pretest, which makes it difficult to evaluate the efficacy of the training task. Future research should continue to focus on developing alternative training techniques that can target the mechanism(s) responsible for attentional biases in anxious individuals.
\end{abstract}

Keywords: Anxiety; Attentional bias; Disengagement from threat; Cueing task

\section{Introduction}

A great deal of effort over the last 30 years has gone into the exploration of how attentional biases relate to or influence anxiety disorders. Numerous studies have demonstrated the presence of attentional biases in emotional disorders [1-3]. These studies, along with many others, have led to the development of cognitive models of anxiety, which presume the notion that biased information processing perpetuates anxiety symptoms in that anxious individuals are typically less inclined to focus on information that indicates neutrality or safety $[4,5]$. What subsequently results is a greater attentional bias for negative or threat-related information in high trait anxious individuals when compared to non-anxious individuals [6,7]. Moreover, recent research has provided evidence that anxiety may result from a failure to disengage one's attention away from threat-related stimuli Fox Russo [8], as opposed to a biased shift in attention toward the threat.

The three most common paradigms used to investigate anxiety related attention biases are: the dot probe, emotional Stroop, and spatial cueing task [6]. Experiments utilizing the dot probe task have shown that anxious individuals, but not nonanxious individuals, are faster at identifying probes that appear in the same locate previously occupied by a threatening stimuli compared to neutral stimuli [7,9]. For the emotional Stroop task, anxious participants have been shown to be slower at naming the color of threat compared to neutral words; however, no difference is found for non-anxious participants [10-12]. While in the cueing task, anxious participants compared to non-anxious participants have been shown to be slower at identifying targets following invalid threat than invalid neutral cues (cue and target appear in different spatial locations) and no difference is found for valid cues (cue and target appear in the same spatial location) $[8,13,14]$.

More recently research has focused on developing attention bias modification techniques aimed at reducing or reversing an individual's attentional bias to threat [15-17]. Utilizing a modified dot probe task, MacLeod [18] have demonstrated that attentional bias towards or away from threat can be trained. Specifically, if participants engage in training trials in which the probe always appears in the same location as the threatening stimuli this can increase their bias towards threat; however, if the probe always appears in the same location as the neutral stimuli this can decrease their bias towards threat. Attention bias modification has been demonstrated to be effective in college students MacLeod [18], individual with sub-clinical ObsessiveCompulsive Disorder Najmi [19], patients with General Anxiety Disorder Amir [20], and general Social Anxiety Disorder [16]. For a full review of recent attention bias modification research see [21]. These interventions have been developed with the hope that 
anxiety would be alleviated through the mitigation of attentional biases to threat. Typically participants not only demonstrate a decrease in their bias towards threat, they also show reduced levels of anxiety symptomatology $[16,20]$.

While it is clear that anxious individuals attend more to threat related information than non-anxious individuals and this bias can be retrained using attention modification techniques, very little research has actually tried to pinpoint which component is responsible for this attentional bias. According to Posner [22], three components are involved in attention: shift, engagement, and disengagement. Koster [23] utilizing the dot probe task response times were compared for trials that contain one threat and one neutral picture followed by a probe that appeared in the same spatial location previous occupied by the neutral picture to trials in which both pictures were neutral followed by a probe that appeared in one or the other spatial locations. A difference score is calculated with a higher positive score indicating delayed disengagement from threat. The results showed delayed disengagement from highly threatening pictures for both anxious and non-anxious individuals; however, when the stimuli are moderately threatening just anxious individuals demonstrated a delayed disengagement from threat. From these results, the authors pointed out that attentional bias to threat can be best accounted for by a problematic disengagement from threat. The rationale for this conclusion is that everybody's attention will be captured by threatening material; regardless of one's trait anxiety. However, it was the moderately threatening stimuli that non-anxious individuals attended to less while the anxious individuals attended to more. Thus, the authors deduced that anxious individuals have a harder time disengaging from threat than non-anxious individuals. This conclusion is consistent with the findings of Georgiou [24] in which anxious participants had longer reaction times compared to non-anxious participants when they were asked to identify a peripheral target while viewing a fearful facial expression located at fixation; indicating that anxious participants took longer to disengage their attention from the threatening stimuli.

Although the above studies have set the groundwork for our current understanding in this area, questions still remain concerning whether or not the underlying cognitive mechanisms are being understood accurately. Given the paradigms that have been employed to study attentional bias, and the following interventions used to reduce that bias, it is still unclear whether anxiety is being perpetuated by a biased shift in attention to threat or an inhibited ability to disengage from threat. Using a dot probe paradigm, it is difficult to make this differentiation. This paradigm presents multiple stimuli to the participant at one time; whereby they are then asked to detect the ensuing probe on an area of the screen where one of the stimuli was just presented. With multiple stimuli presented, one cannot conclusively determine what the underlying attentional processes are. One way to help sort this out is through a cueing paradigm where only one stimulus is presented, after which participants are required to detect a target. Under this paradigm, a cue can be valid; in which case the cue and target appear in the same spatial location.
On the other hand, a cue can be invalid; in which case the cue and target appear in different locations. Having only one cueing stimulus isolates attentional bias to difficulties with attentional disengagement from threat, due to the fact that there are no other stimuli present that a participant could shift their attention to. Otherwise, the potential variables of someone shifting attention between multiple stimuli or the purposeful avoidance of certain stimuli cannot be ruled out. Therefore, differential response latencies to targets, depending on cue valence and validity, will be a matter of attentional disengagement.

The goal of the present study was to fill a gap within the literature by implementing a novel intervention that would modify/decrease attentional bias for threat. Although attentional modification training has used the dot probe paradigm, as cited earlier, little or no research has examined the use of a cueing paradigm to train attention. Using such a paradigm in this manner is important, because problems with attentional disengagement can be isolated and addressed. Whereas presently, it is uncertain whether attentional modification training is decreasing bias toward threat (i.e. attentional shifting) or aiding in one's attentional disengagement from it. As a result, this study used a cueing task aimed to specifically enhance attentional engagement with neutral stimuli by using only valid neutral cues and disengage their attention from threatening stimuli using only invalid threat cues during training. Two training groups (explicit and implicit) and one control group were used in this experiment. All participants completed a pretest and posttest, which consisted of $80 \%$ threatening and neutral valid pictorial cue and $20 \%$ threatening and neutral invalid pictorial cues. During training, both training groups were given only valid neutral and invalid threat cues; while the control group continued to receive all cue types with the same contingency that was used in the pretest. In the explicit training group, participants were made aware of this new contingency while in the implicit group they were not.

For pretest trials, it was first hypothesized that valid cues would be detected faster than invalid cues (H1). Secondly, it was expected that an invalid threat cue would cause slower responses to the target when compared to the invalid neutral cue; however there should be no difference in response latency between valid threat and valid neutral cues (H2). This should provide evidence for the presence of problematic disengagement from threat rather than a bias towards a rapid shift of attention to threat.

Due to training, participants in the training groups should come to expect that all neutral cues will be valid and therefore, show an enhanced engagement with and delayed disengagement from neutral cues compared to control participants who should expect that the majority, but not all, neutral cues to be valid. Participants in the explicit training group received specific verbal instructions regarding the cue contingency (neutral cues always valid and threat cues always invalid). Given this level of conscious awareness, the explicit group should show a greater effect of training on performance compared to the implicit group. Therefore for posttest trials, it was thirdly hypothesized that the magnitude of difference between valid neutral and invalid neutral cues would be greatest for the explicit group, followed by 
the implicit group, and then the control group (H3). Likewise due to training, participants in the training groups should come to expect that all threatening cues will be invalid and should show a rapid disengagement from threatening cues compared to control participants who should expect that the majority of threatening cues will be valid. Therefore, it was fourthly predicted that the magnitude of difference in detecting invalid threat compared to valid threat cues would be smaller for the explicit training, followed by implicit training, and then the control group (H4).

Lastly, it was expected that the training groups would experience a significant decrease in state anxiety from pretest to posttest relative to the control group due to a decrease in engagement with the threatening material as a result of training (H5).

\section{Method}

\section{Participants}

Eighty-nine undergraduate students from Biola University were recruited for the present study. They received course credit, extra credit, or the chance to win a $\$ 25$ gift-card/prize through a raffle drawing as compensation for their participation. In order to test only high trait anxious participants, individuals who scored at or above 45 on the trait portion Spielberger State-Trait Anxiety Inventory Spielberger [25] were pre-selected to take part in the study. This inventory was completed in their classes prior to participation. Ethical approval was obtained from the Protection of Human Rights in Research Counsel at Biola University.

\section{Materials and apparatus}

In order to run the cueing task and record participants' response latencies to targets, Dell Optiplex 755 computers using the program E-Prime 2.0 Schneider [26] were utilized. Computer monitors $(28 \mathrm{~cm} \times 43 \mathrm{~cm})$ with a black background displayed either a neutral or threatening pictorial cue in one of four possible spatial locations on the screen around a white fixation cross (+) at the center. Cues and targets were presented within a $20 \mathrm{~cm}$ x $20 \mathrm{~cm}$ area centered on the monitor. These cues could appear either above, below, to the left, or to the right of the fixation. With participants sitting approximately $75 \mathrm{~cm}$ away from the screen, cues occupied $6^{\circ}$ of visual angle. The distance between the fixation and the edge of the cues was $6^{\circ}$ of visual angle.

Ten threatening $(6550,6560,6260,3110,3016,3195,3530$, 6520,6570 , and 9910) and 10 neutral $(2512,7090,7100,7130$, $7150,7170,7235,7500,7550$, and 2396) pictures were selected from the International Affective Pictures System Lang [27] were used as cues. Independent samples t-test showed the threatening pictures were significantly more unpleasant/negative $(\mathrm{M}=2.11$, $\mathrm{SD}=0.29)$ than the neutral pictures $(\mathrm{M}=5.04, \mathrm{SD}=0.22), t(9)$ $=-25.19, \mathrm{p}<.001$. Threatening pictures also had significantly higher ratings of arousal $(\mathrm{M}=6.53, \mathrm{SD}=0.38)$ compared to neutral pictures $(M=3.15, S D=0.42), t(9)=18.82, p<.001$.

The target, capital letters X or P using "Courier New" font, appeared centered in any of the four spatial locations on the screen. When an "X" was detected, participants responded by pressing the "Z" key on the keyboard in front of them. When the "P" was detected, they responded by pressing the "M" key. Each target occupied $1.3^{\circ}$ of visual angle. The distance between the fixation and the edge of the target was $8^{\circ}$ of visual angle. Furthermore, the fixation occupied $1^{\circ}$ of visual angle.

State portion of the Speilberger State-Trait Anxiety Inventory (STAI-S) and the Beck Depression Inventory Beck [28] were administered to each participant.

\section{Procedure}

Participants were randomly assigned to the explicit training, implicit training, or control group. One-way between-subjects ANOVAs revealed that there were no significant differences between the three groups regarding trait anxiety, $F(2,86)=2.14$, $p=0.12$, or depression, $F(2,86)=1.15, p=0.32$ ( Table 1 for means, standard errors, and 95\% CI). First, participants were assessed regarding their state anxiety using the STAI-S. Next, computerized instructions made participants aware of their task depending on which group they were assigned to. Each trial consisted of the presentation of the fixation cross, a cue, and a target. After the fixation appeared on the screen for $1000 \mathrm{~ms}$, a cue was presented for $500 \mathrm{~ms}$. After its termination, $1000 \mathrm{~ms}$ elapsed before a target appeared. The target appeared centered in any of the four spatial locations and remained on the screen for $2000 \mathrm{~ms}$ or until a participant responded followed by an intertrial-interval of $1000 \mathrm{~ms}$.

During a valid trial the target appeared in the same spatial location as the cue, while during an invalid trial the target appeared in one of the other three possible spatial locations where the cue was not presented. The valence (threat, neutral) and cue type (valid, invalid), as well as their spatial location (i.e., above, below, to the left, or to the right of the fixation), were randomly presented on the screen.

For the pretest, all groups experienced a preset contingency of valid to invalid trials; such that $80 \%$ of the trails were valid while the remaining $20 \%$ were invalid, with an equal number of neutral and threat cues being presented. After the pretest, the two training groups completing training trials in which there was a $100 \%$ contingency between a neutral cue and the target that replaced its position on the screen. In other words, all neutral cues were valid. On the other hand, there was a $0 \%$ contingency between the threat cue and the target position. Here, all threat cues were invalid. The explicit training group received very direct computerized instructions alerting them to this contingency; whereas, the implicit group received no such instruction. The control group continued to receive the same contingency used during the pretest. Lastly, a posttest identical to the pretest was completed by all three groups followed by each participant completing the STAI-S and BDI. There were 200 pretest, 300 training, and 200 posttest trials and the entire experiment lasted 1 hour 15 minutes.

\section{Data analysis}

Reaction time data was filtered by removing inaccurate trials along with trials in which reaction times were greater than 2000 
Table 1: Depression and Trait Anxiety Levels Among Participants by Group.

\begin{tabular}{|l|l|l|l|l|l|l|l|l|l|}
\hline \multicolumn{3}{|l}{ Control } & \multicolumn{3}{l}{ Implicit } & \multicolumn{2}{l|}{ Implicit } \\
\hline Measure & $\mathrm{n}$ & $\mathrm{M}(\mathrm{SE})$ & $95 \%$ CI & $\mathrm{n}$ & $\mathrm{M}(\mathrm{SE})$ & $95 \%$ CI & $\mathrm{n}$ & M (SE) & $95 \%$ CI \\
\hline BDI & 29 & $12.8(1.5)$ & {$[9.7,16.0]$} & 30 & $14.6(1.4)$ & {$[11.7,17.5]$} & 30 & $16.0(1.5)$ & {$[12.9,19.1]$} \\
\hline STAI-Trait & 29 & $48.0(1.4)$ & {$[45.3,50.8]$} & 30 & $48.9(1.0)$ & {$[46.8,1.0]$} & 30 & {$[49.0,53.8]$} \\
\hline
\end{tabular}

Note: CI = confidence interval. The Beck Depression Inventory (BDI) is from [26]; the Spielberger State-Trait Anxiety Inventory - Trait version (STAI - Trait) is from [24].

ms or less than $200 \mathrm{~ms}$. Exploratory analysis was then conducted on the reaction time data using a 2 (valence: threat, neutral) $\mathrm{x}$ 2 (cue type: valid, invalid) x 3 (group: explicit, implicit, control) x 2 (time: pretest, posttest) mixed design ANOVA. Furthermore, specific analyses were conducted to test the five hypotheses. Paired-samples t-test was utilized to determine if at pretest, valid cues were detected faster than invalid cues (H1), invalid neutral cues were detected faster than invalid threat cues, and whether there was no difference between valid neutral and valid threat cues (H2). For the posttests, it was expected that the magnitude of difference between valid and invalid neutral cues (H3) and valid and invalid threat cues (H4) would differ according to training condition (explicit, implicit, control); therefore, difference scores were calculated by subtracting reaction times for valid minus invalid trials. Then independent samples t-tests were performed on the difference scores comparing the three groups. A 2 (time: pretest, posttest) x 3 (group: explicit, implicit, control) mixed factorial ANOVA was conducted to test hypothesis 5 that the level of state anxiety would decreased from pretest to posttest for the training groups, but not the control group. As there were no specific hypotheses regarding the accuracy, the accuracy data was analyzed using 2 (valence: threat, neutral) $x$ 2 (cue type: valid, invalid) x 3 (group: explicit, implicit, control) x 2 (time: pretest, posttest) mixed design ANOVA. All significant interactions from the ANOVA analyses were followed-up using simple main effects analysis.

\section{Results}

\section{Reaction time}

Exploratory analysis: Results of the exploratory mixed factorial ANOVA (Table 2 for means and standard errors) showed that there was a significant main effect for cue type, $F(1,86)=$ 86.85, $p<0.001, \eta_{P}^{2}=0.50$, such that participants were faster at detecting valid cues compared to invalid cues. The results also showed the presence of a main effect for group, $F(2,86)=3.84$, $p=0.025, \eta_{P}^{2}=0.08$. Post hoc comparisons using the Fisher LSD test revealed that participants in the explicit group responded significantly slower to targets than the control group, Mean difference $=60, p=0.027$. Furthermore, the explicit group was also significantly slower than the implicit group, Mean difference $=67, p=0.013$. Lastly, no significant main effects for valence or time were observed, $F^{\prime} s(1,86)<0.871, p^{\prime} s>0.353$. Analyses did not show the presence of a time $\mathrm{x}$ cue type $\mathrm{x}$ group $\mathrm{x}$ valence interaction in the data, $F(2,86)=1.14, p=0.32$. Likewise, none of the other interactions were significant, $F^{\prime} s(2,86)<2.16, p^{\prime} s>0$ .146; except for a trend in the valence $\mathrm{x}$ group interaction, $F(2$, 86) $=2.51, p=0.087$.

\section{Specific hypotheses}

Pretest data hypotheses 1 and 2: The results of paired samples t-tests showed that at pretest valid cues were detected significantly faster than invalid cues for all three groups: explicit, $t(29)=-3.89, p=0.001$; implicit, $t(29)=-5.42, p<0.001$, and control $t(28)=-5.19, p<0.001$ (H1). However, paired sampled t-tests comparing valid threat and valid neutral cues (H2) were not significant for any of the three groups, $t^{\prime} s<1.01, p^{\prime} s>$ 0.321 ; indicating no attentional shift bias towards threatening stimuli. Likewise, paired samples t-tests comparing invalid threat and invalid neutral pretest trials (H2) were not significant for the control and explicit groups, $t(28)=0.63, p=0.535$ and $t(29)=-0.77, p=0.450$, respectively; indicating no attentional disengagement bias for threatening stimuli. The result was significant for the implicit group, $t(29)=2.12, p=0.043$. Contrary to prediction participants were faster during invalid threat compared to invalid neutral trials indicating a bias away from threat.

Posttest data hypotheses 3 and 4: The results of independent samples t-tests on difference scores (valid - invalid) for the neutral cues (H3) showed a significant difference between the explicit and control group, $t(57)=2.21, p=0.031$, with a larger mean difference found for the explicit (Mean difference $=$ -55.67) compared to control group (Mean difference $=-23.61$ ) . However, neither the explicit vs. implicit, $t(58)=-1.17, p=0.245$, or the control vs. implicit, $t(57)=1.09, p=0.282$, comparisons were significant. Similarly, results from independent samples t-tests on the difference scores for threatening cues (H4) showed that none of the comparisons were significant, $t$ 's $<1, p$ 's $>0.346$.

\section{Anxiety results}

Hypothesis 5: Results from a 2 (time: pre-state, post-state) $\mathrm{x}$ 3 (group: explicit, implicit, control) mixed factorial ANOVA (Table 3 for means and standard errors) showed that the main effect for group was not significant, $F(2,86)=0.480, p=0.621$, indicating that state anxiety levels were comparable between groups. Yet, state anxiety scores at posttest were significantly higher than state scores at pretest, thus resulting in a significant main effect for time, $F(1,86)=4.08, p=0.047, \eta_{P}^{2}=0.05$. Furthermore, a time $\mathrm{x}$ group trend was also found, $F(2,86)=3.02, p=0.054$.

Based on the a priori hypotheses, and the trend towards a significant interaction between time and group, simple main effects analysis for time was performed for each group. For the implicit group, no significant changes in state anxiety were observed from pretest to posttest, $F(1,86)=0.70, p=0.404$. On the other hand, the explicit group did experience a statistically 
Table 2: Mean (SE) Reaction Times of Participants by Group in Milliseconds.

\begin{tabular}{|c|c|c|c|c|c|c|c|c|c|c|c|c|}
\hline \multicolumn{13}{|l|}{ Group } \\
\hline \multirow[b]{2}{*}{ Condition } & \multicolumn{4}{|l|}{ Control } & \multicolumn{4}{|l|}{ Explicit } & \multicolumn{4}{|l|}{ Implicit } \\
\hline & Neutral & $\mathrm{M}(\mathrm{SE})$ & Threat & $\mathrm{M}(\mathrm{SE})$ & $\begin{array}{l}\text { Neutral } \\
\text { (SE) }\end{array}$ & M & Threat & $M(S E)$ & $\begin{array}{l}\text { Neutral } \\
\text { (SE) }\end{array}$ & M & Threat & $\mathrm{M}(\mathrm{SE})$ \\
\hline Pretest & \multirow{2}{*}{\multicolumn{2}{|c|}{519 (18) }} & \multirow{2}{*}{\multicolumn{2}{|c|}{519 (19) }} & \multirow{2}{*}{\multicolumn{2}{|c|}{573 (18) }} & \multirow{2}{*}{\multicolumn{2}{|c|}{573 (18) }} & \multirow{2}{*}{\multicolumn{2}{|c|}{516 (18) }} & \multirow{2}{*}{\multicolumn{2}{|c|}{512 (18) }} \\
\hline Valid & & & & & & & & & & & & \\
\hline Invalid & $555(20)$ & & 551 (19) & & $606(20)$ & & $613(18)$ & & $557(20)$ & & 541 (18) & \\
\hline Posttest & \multirow{2}{*}{\multicolumn{2}{|c|}{515 (22) }} & \multirow{2}{*}{\multicolumn{2}{|c|}{518 (23) }} & \multirow{2}{*}{\multicolumn{2}{|c|}{564 (21) }} & \multirow{2}{*}{\multicolumn{2}{|c|}{575 (23) }} & \multirow{2}{*}{\multicolumn{2}{|c|}{$502(21)$}} & \multirow{2}{*}{\multicolumn{2}{|c|}{502 (23) }} \\
\hline Valid & & & & & & & & & & & & \\
\hline Invalid & 539 (23) & & 537 (23) & & $620(22)$ & & 613 (22) & & $540(22)$ & & $530(22)$ & \\
\hline
\end{tabular}

Table 3: State Anxiety levels Among Participants by Group at Pretest and Posttest.

\begin{tabular}{|l|l|l|l|l|l|l|l|l|l|}
\hline \multicolumn{3}{|l}{ Control } & \multicolumn{3}{l}{ Explicit } & \multicolumn{3}{l|}{ Implicit } \\
\hline Measure & n & M (SE) & $\mathbf{9 5 \%}$ CI & n & M (SE) & $\mathbf{9 5 \%}$ CI & n & M (SE) & $\mathbf{9 5 \%}$ CI \\
\hline $\begin{array}{l}\text { Pretest } \\
\text { STAI-State }\end{array}$ & 29 & $40.6(2.0)$ & {$[36.6,44.8]$} & 30 & $39.9(1.9)$ & {$[36.1,43.7]$} & 30 & $44.4(1.9)$ & {$[40.7,48.2]$} \\
\hline $\begin{array}{l}\text { osttest } \\
\text { STAI-State }\end{array}$ & 29 & $43.9(1.5)$ & {$[40.8,47.1]$} & 30 & $43.2(2.0)$ & {$[39.1,47.3]$} & 30 & $43.2(1.7)$ & {$[39.8,46.6]$} \\
\hline
\end{tabular}

Note: $\mathrm{CI}$ = confidence interval. The Spielberger State-Trait Anxiety Inventory - State version (STAI - State) is from [24].

Table 4: Mean (SE) Accuracy Rates of Participants' Responses to Targets.

\begin{tabular}{|c|c|c|c|c|c|c|}
\hline \multicolumn{7}{|l|}{ Group } \\
\hline \multirow[b]{2}{*}{ Condition } & \multicolumn{2}{|l|}{ Control } & \multicolumn{2}{|l|}{ Explicit } & \multicolumn{2}{|l|}{ Implicit } \\
\hline & Neutral M (SE) & Threat M (SE) & Neutral M (SE) & Threat M (SE) & Neutral M (SE) & Threat M (SE) \\
\hline Pretest & \multirow{2}{*}{$0.97(0.01)$} & \multirow{2}{*}{$0.97(0.01)$} & \multirow{2}{*}{$0.97(0.01)$} & \multirow{2}{*}{$0.97(0.01)$} & \multirow{2}{*}{$0.97(0.01)$} & \multirow{2}{*}{$0.98(0.01)$} \\
\hline Valid & & & & & & \\
\hline Invalid & $0.96(0.01)$ & $0.98(0.01)$ & $0.97(0.01)$ & $0.96(0.01)$ & $0.98(0.01)$ & $0.98(0.01)$ \\
\hline Posttest & \multirow{2}{*}{$0.97(0.01)$} & \multirow{2}{*}{$0.99(0.01)$} & \multirow{2}{*}{$0.95(0.01)$} & \multirow{2}{*}{$0.95(0.01)$} & \multirow{2}{*}{$0.98(0.01)$} & \multirow{2}{*}{$0.97(0.01)$} \\
\hline Valid & & & & & & \\
\hline Invalid & $0.96(0.01)$ & $0.97(0.01)$ & $0.96(0.01)$ & $0.94(0.01)$ & $0.97(0.01)$ & $0.98(0.01)$ \\
\hline
\end{tabular}

significant change in their anxiety, $F(1,86)=4.78, p=0.032, \eta_{P}^{2}$ $=0.05$, yet in the opposite direction than what was anticipated, such that anxiety increased from pretest to posttest. In addition, the control group showed an increase in state anxiety at posttest compared to pretest, $F(1,86)=4.55, p=0.036, \eta_{P}^{2}=0.05$.

\section{Accuracy}

A final set of analyses employed a 2 (valence: threat, neutral) x 2 (cue type: valid, invalid) x 3 (group: explicit, implicit, control) x 2 (time: pretest, posttest) mixed design ANOVA to examine the differences in participants' accuracy when responding to targets (Table 4 for means and standard errors). The results showed a significant main effect for time, $F(1,86)=5.99, p=0.016, \eta_{P}^{2}=$ 0.07 , such that participants were less accurate at posttest than pretest. A significant main effect for group was also demonstrated, $F(2,86)=3.46, p=0.036, \eta_{p}^{2}=0.07$. Post hoc comparisons using the Fisher LSD test demonstrated that the explicit group was significantly less accurate than the implicit group, Mean difference $=0.017, p=0.015$, with a trend for the control group, Mean difference $=0.014, p=0.052$. The main effects for valence and cue type were not significant, $F^{\prime} S(1,86)<0.377, p$ ' $s>.541$.

There was a trend towards a significant time $\mathrm{x}$ group $\mathrm{x}$ cue type $\mathrm{x}$ valence interaction emerged, $F(2,86)=2.75, p=0.070$. In addition, a significant time $\mathrm{x}$ cue type interaction was found, $F(2,86)=4.06, p=0.047, \eta_{p}^{2}=0.05$. Simple main effects analyses showed that accuracy for invalid cues was significantly higher during pretest compared to posttest, $F(1,86)=7.61, p=0.007$, $\eta_{P}^{2}=0.08$; whereas, there was no significant difference between pretest and posttest valid cues, $F(1,86)=0.93, p=0.338$. None of the other interactions were significant, $F^{\prime} s(2,86)<2.25$, $p^{\prime} s>$ 0.111 .

\section{Discussion}

The purpose of the present study was to implement a novel intervention that would modify/decrease trait anxious participants' attentional biases for threatening stimuli. Through the use of a modified cueing task it was predicted that participants' state anxiety would subsequently decrease through a shift in their attentional engagement with neutral and disengagement from threatening photographs. As evidenced by the results, many of the hypotheses in this study regarding response times were not supported. However, two fundamental hypotheses were supported. In congruence with past research in this field Fox $[8,13,14,29]$, the validity of the cue impacted the participants' response to the targets in such a way that valid cues 
were detected faster than invalid cues both overall and more specifically during pretest trials (H1). In other words, because participants' attention was already drawn to a particular spatial location on the screen, it was more likely that a target would be detected faster if it appeared in that same location. This finding supports the concept that regardless of the nature of the stimuli, cue validity plays an important role in one's reaction time to targets. Furthermore, similar to previous literature Fox $[8,13,14]$, there were no significant differences in response time during pretest between valid threat and valid neutral cues.

Yet, with regards to the hypotheses unique to the present study (i.e., greater attentional biases toward threat at pretest and enhanced attentional disengagement from threat among the training groups at posttest), little support was found. Specifically, an attentional bias in anxious participants towards threat at pretest was not observed. Contrary to the author's expectations, invalid threat cues were detected no slower than invalid neutral cues. In previous research by Fox $[8,13]$, anxious individuals showed a pronounced delay in attentional disengagement from invalid threat cues than with invalid neutral cues; however, this pattern was not observed in the present study. In fact, the implicit group at pretest was actually faster at detecting invalid threat cues compared to invalid neutral cues. This attentional bias was in the opposite direction as hypothesized, such that they rapidly disengaged from the invalid threat cues despite no training. One reviewer suggested that this failure to replicate a delayed disengagement from threat may have been due to participants' level of trait anxiety - perhaps too low for a bias to be detected. However, the current study used a trait anxiety cut off of 45 , which is higher than that used in Fox $[8,13]$ studies that utilized a cut off score of 40 and therefore should have been high enough to detect anxiety related attentional biases. The same reviewer also suggested that perhaps based on the findings of Verkuil [30] that it is only when high trait anxiety coincides with high trait worry that attentional disengagement biases are observed. This seems like a more plausible explanation in that attentional biases towards threat could be moderated by additional personality factors, such as worry, and that only under a certain combination of these factors that one's attention is biased towards threatening stimuli. It should be noted, however, that Verkuil et al. found that participants who were scored high on both anxiety and worry showed a rapid disengagement from invalid threat compared to valid threat cues indicating an inhibition of return Posner [31] rather than a delayed disengagement from threat.

Regarding the hypothesized pretest to posttest differences in response times to valid threat and invalid threat trials, training did not appear to affect participants' responses in the expected direction. Contrary to this study's hypotheses, training did not result in faster response latencies for invalid threat cues at posttest for the explicit and implicit groups, when compared to both their pretest times and controls. Additionally, while it was anticipated that training would increase one's response times for valid threat trials at posttest for the training conditions, the results did not show such a difference. The presence of slower responses to valid threat cues and faster responses to invalid threat cues at posttest would have provided evidence that the attentional training used in this study helped participants disengage their attention from threat. Furthermore, it was hypothesized that the training groups' detection of targets following valid neutral cues would be faster than invalid neutral cues when compared to controls due to training. With greater attentional engagement with neutral cues (and their spatial location) during training, it was hoped that this training would have carried over into the posttest. In support of this hypothesis, the explicit training group evidenced a larger mean difference in response times than the control group - indicating that training led to a greater magnitude of difference compared to the difference observed when no training was employed. With regards to the two training groups, it was initially hypothesized that the group receiving explicit training would have shorter response latencies to valid neutral cues and invalid threat cues than the implicit training group. Again the data did not support this finding. However, given the lack of an initial attentional bias towards threat during pretest, in fact the implicit group showed a bias away from threat, it is not surprising that there was then no subsequent decrease observed at posttest.

As for participants' anxiety levels, it was expected that the training groups would experience a significant decrease in state anxiety relative to the control group due to a decrease in engagement with the threatening material as a result of training. The implicit group showed no difference in anxiety at posttest; however, contrary to expectations, the explicit group experienced a statistically significant increase in anxiety. A plausible explanation is that the presence of explicit instructions to modify attentional engagement may have created a cognitively demanding and stressful situation. Such instructions during training may have necessitated the use of higher level cognitive processing to complete the task. Since participants were already being presented with various images in rapid succession, additional information to keep in mind to complete the task may have led to greater levels of arousal, which in turn resulted in higher anxiety. Consequently, this increase in load may have also impacted the explicit group's response latencies. Given the explicit group's slower responses and higher error rate, in comparison to the implicit and control group, higher levels of anxiety may have impaired their attentional processes to the extent that making rapid attentional shifts may have been more difficult. This is consistent with previous findings by Berggren [32], which showed that high working memory load impaired anxious individuals from disengaging their attention from angry facial expressions. It should be noted again that compared to the other two groups, who did show an increase in anxiety - the implicit training group did not. Although their anxiety did not decrease as expected, it at least stayed the same, which may be an indication that the training did provide some protection against the stress of the study.

With regards to the accuracy data, participants as a whole were less accurate at posttest than pretest. Further analyses revealed that this result was primarily due to the explicit group not only being less accurate than the implicit and control groups, but also being less accurate during posttest compared to pretest 
trials. With these findings in mind, it may be plausible to assume that the increase in these participants' anxiety influenced their reaction time at posttest, in addition to negatively impacting their accuracy. This observation would be consistent with anxiety's tendency to have a negative/inhibitory effect on people when they are required to perform precision tasks under the pressure of time [33]. Furthermore, having to concurrently keep explicit instructions in mind when making a key press contingent upon the target letter may have required a great deal of mental energy. Maintaining such focus for an extended period of time could have been both stressful and fatiguing.

A key difference in methodology between that employed in the present study and previous research was the use of four possible spatial location instead of two $[8,13]$. This may have made the task unnecessarily more complex and masked any attentional biases that may have been present. Additionally, a reviewer suggested that perhaps the pictures were not threatening enough to elicit delayed disengagement. Although Koster et al. [23] found a disengagement bias for both moderate/ mild and high threat IAPS pictures using the dot probe paradigm, Koster [34] using what appears to be the same IAPS pictures in a cueing paradigm found a delayed disengagement just for high threat, but not moderate/mild threat pictures. The pictures used in the current study comprised moderately threatening IAPS pictures. Perhaps within the cueing paradigm it may be essential to utilize highly threatening stimuli in order to elicit attentional biases. So although there was no evidence for the efficacy of using a modified cueing task to retrain attentional biases a simpler paradigm that just utilizes two spatial locations and highly threatening stimuli may be a promising area for future research.

In conclusion, this study did not find support for the use of a modified cueing task in retraining attentional bias; however, given the lack of attentional biases at pretest one cannot rule out the possibility that this could be an effective paradigm. One advantage that this paradigm has over the dot probe task is that training could be directed specifically at attentional engagement with neutral stimuli, disengage with threatening stimuli, or both as used here. Given these possible advantages, future research in this area is warranted. It is recommended that the modified cueing task be further developed; specifically utilizing implicit training with two spatial locations rather than four. In addition, training could solely target just one mechanism of attention either engagement or disengagement. Lastly, previous cognitive bias modification studies that have used multiple training sessions have demonstrated more promising results in their ability to reduce a cognitive/attentional bias toward threat [16]. Subsequent intervention studies that employ a cueing paradigm may benefit from using multiple training sessions.

\section{References}

1. Beck AT, Clark DA (1997) An information processing model of anxiety: Automatic and strategic processes. Behav Res Ther 35(1): 49-58.

2. Mathews A, MacLeod C (1994) Cognitive approaches to emotion and emotional disorders. Annu Rev Psychol 45: 25-50.

3. Mogg K, Bradley BP (1998) A cognitive-motivational analysis of anxiety. Behav Res Ther 36(9): 809-848.

4. Mathews A (1990) Why worry? The cognitive function of anxiety. Behav Res Ther 28(6): 455-468.

5. Mathews A, MacLeod C (2002) Induced processing biases have causal effects on anxiety. Cognition and Emotion 16(3): 331-354.

6. Bar Haim Y, Lamy D, Pergamin L, Bakermans Kranenburg MJ, van Ijzendoorn, MH (2007) Threat-related attentional bias in anxious and non-anxious individuals: A meta-analytic study. Psychol Bull 133(1): $1-24$.

7. MacLeod C, Mathews A, Tata P (1986) Attentional bias in emotional disorders. J Abnorm Psychol 95(1): 15-20.

8. Fox E, Russo R, Bowles R, Dutton K (2001) Do threatening stimuli draw or hold visual attention in subclinical anxiety? J Exp Psychol Gen 130(4): 681-700

9. Bradley BP, Mogg K, White J, Groom C, de Bono J (1999) Attentional bias for emotional faces in generalized anxiety disorder. Br J Clin Psychol 38(3): 267-278.

10. Bradley BP, Mogg K, Miller N, White J (1995) Selective processing of negative information: Effects of clinical anxiety, concurrent depression, and awareness. J Abnorm Psychol 104(3): 532-536.

11. Mathews A, MacLeod C (1985) Selective processing of threat cues in anxiety states. Behav Res Ther 23(5): 563-569.

12. Mogg K, Mathews A, Weinman J (1989) Selective processing of threat cues in anxiety states: A replication. Behav Res Ther 27(4): 317-324.

13. Fox E, Russo R, Dutton K (2002) Attentional bias for threat: Evidence for delayed disengagement from emotional faces. Cogn Emot 16(3): 355-379.

14. Yiend J, Mathews A (2001) Anxiety and attention to threatening pictures. Q J Exp Psychol A 54(3): 665-681.

15. MacLeod C, Koster EHW, Fox E (2009) Whither cognitive bias modification research? Commentary on the special section articles. J Abnorm Psychol 118(1): 89-99.

16. Schmidt NB, Richey JA, Buckner JD, Timpano KR (2009) Attention training for generalized social anxiety disorder. J Abnorm Psychol 118(1): 5-14

17. See J, MacLeod C, Bridle R (2009) The reduction of anxiety vulnerability through the modification of attentional bias: A real-world study using a home-based cognitive bias modification procedure. J Abnorm Psychol 118(1): 65-75.

18. MacLeod C, Rutherford E, Campbell L, Ebsworthy G, Holker L (2002) Selective attention and emotional vulnerability: Assessing the causal basis of their association through the experimental manipulation of attentional bias. J Abnorm Psychol 111(1): 107-123.

19. Najmi S, Amir N (2010) The effect of training attention on a behavioral test of contamination fears in individuals with subclinical obsessivecompulsive symptoms. J Abnorm Psychol 119(1): 136-142.

20. Amir N, Beard C, Burns M, Bomyea J (2009) Attention modification program in individuals with generalized anxiety disorder. J Abnorm Psychol 118(1): 28-33.

21. Bar Haim Y (2010) Research review: Attention bias modification (ABM): a novel treatment for anxiety disorders. J Child Psychol Psychiatry 51(8): 859-870.

22. Posner M I, Petersen S E (1990) The attention system of the human brain. Annu Rev Neurosci 13: 24-42. 
23. Koster EHW, Crombez G, Verschuere B, De Houwer J (2006) Attention to threat in anxiety-prone individuals: Mechanism underlying attentional bias. Cognitive Therapy and Research 30(5): 635-643.

24. Georgiou A, Bleakley C, Hayward J, Russo R, Dutton K, Eltiti S, Fox E (2005) Focusing on fear: Attentional disengagement from emotional faces. Vis cogn 12(1): 145-158.

25. Spielberger CD, Gorsuch RL, Lushene R, Vagg PR, Jacobs GA (1983) Manual for the State-Trait Anxiety Inventory. Consulting Psychologists Press, Palo Alto, California, USA.

26. Schneider W, Eschman A, Zuccolotto A (2002) E-Prime User's Guide. Psychology Software Tools Inc. Pittsburgh, Pennsylvania, USA

27. Lang PJ, Bradley MM, Cuthbert BN (2008) International affective picture system (IAPS): Affective ratings of pictures and instruction manual. Technical Report A-8, University of Florida, Gainesville, FL, USA.

28. Beck AT, Steer RA, Brown GK (1996) Beck depression inventory. (2nd edn), Psychological Corporation, San Antonio, Texas, USA.
29. Posner MI (1980) Orienting of attention. Q J Exp Psychol 32(1): 3-25.

30. Verkuil B, Brosschot JF, Putman P, Thayer JF (2009) Interacting effects of worry and anxiety on attentional disengagement from threat. Behav Res Ther 47(2): 146-152.

31. Posner MI, Cohen Y (1984) Components of visual orienting. In: $\mathrm{H}$. Bouma, DG Bouwhuis (Eds.), Attention and performance X: Control of language processes Erlbaum, Hillsdale, New Jersey, USA, pp. 531-556.

32. Berggren N, Koster EHW, Derakshan N (2012) The effect of cognitive load in emotional attention and trait anxiety: An eye movement study. Journal of Cognitive Psychology 24(1): 79-91.

33. Eysenck MW, Calvo MG (1992) Anxiety and performance: The processing efficiency theory. Cognition and Emotion 6(6): 409-434.

34. Koster EHW, Crombez G, Verschuere B, Van Damme S, Roelf Wiersema $J$ (2006) Components of attentional bias to threat in high trait anxiety: Facilitated engagement impaired disengagement and attentional avoidance. Behaviour Research and Therapy 44(12): 1757-1771. 